\title{
Efeito de doses de paclobutrazol na cultura do alho(1)
}

\author{
Geraldo Milanez de Resende( ${ }^{(2)}$ e Rovilson José de Souza(3)
}

\begin{abstract}
Resumo - O trabalho foi realizado no período de maio a outubro de 1991 no campo experimental do setor de olericultura da Ufla, Lavras, MG, com o objetivo de avaliar a influência de doses de paclobutrazol sobre o controle do pseudoperfilhamento e nas características morfológicas e comerciais do alho (Allium sativum L.). Utilizou-se o delineamento experimental de blocos ao acaso, com quatro doses de paclobutrazol $\left(0,500,1.000\right.$ e $1.500 \mathrm{mg}$ de i.a. $\left.\mathrm{L}^{-1}\right)$, em cinco repetições. Com o aumento das concentrações de paclobutrazol, houve uma redução na altura das plantas e no número de folhas por planta aos 60 e 90 dias após o plantio. A produtividade total e comercial de bulbos apresentou efeito significativo em relação às doses de paclobutrazol, sendo as concentrações de 725 e $778 \mathrm{mg} \mathrm{L}^{-1}$ as que proporcionaram as maiores produtividades. A porcentagem de bulbos pseudoperfilhados evidenciou efeito quadrático com o incremento das doses de paclobutrazol, cuja concentração de $1.163 \mathrm{mg} \mathrm{L}^{-1}$ propiciou maior redução na porcentagem de pseudoperfilhamento. A concentração de $744 \mathrm{mg} \mathrm{L}^{-1}$ de paclobutrazol proporcionou o maior peso médio de bulbo; e em relação a número de bulbilhos por bulbo, não se verifícaram diferenças significativas entre os tratamentos.
\end{abstract}

Termos para indexação: Allium sativum, substâncias de crescimento vegetal, rendimento, produtividade.

\section{Effects of paclobutrazol doses on garlic crop}

\begin{abstract}
This study was carried out at the experimental field of Universidade Federal de Lavras, Minas Gerais State, Brazil, from May to October, 1991, and aimed at evaluating the influence of paclobutrazol doses on secondary growth control and morphologic and marketable characteristics of garlic (Allium sativum L.). The experimental design was a randomized complete block with five replications and four paclobutrazol doses $\left(0,500,1,000\right.$ and 1,500 $\mathrm{mg}$ of a.i. $\left.\mathrm{L}^{-1}\right)$. Reduction in plant height and in number of leaves per plant at 60 and 90 days after planting was observed with paclobutrazol applications. Total bulbs and marketable yields showed significant effects with the application of paclobutrazol doses, and both concentrations of $725 \mathrm{mg} \mathrm{L}^{-1}$ and $777 \mathrm{mg} \mathrm{L}^{-1}$ were the ones with best performance. The percentage of secondary growth evidenced negative quadratic effect with the increment of paclobutrazol doses, and the concentration of $1,163 \mathrm{mg} \mathrm{L}^{-1}$ showed the highest reduction. Mean bulb weight increased up to the dose of $744 \mathrm{mg} \mathrm{L}^{-1}$ of paclobutrazol. There were no significant differences among treatments for cloves per bulb.
\end{abstract}

Index terms: Allium sativum, plant growth substance, yields, productivity.

\section{Introdução}

Os reguladores de crescimento, em razão de seus efeitos sobre diferentes processos fisiológicos das

\footnotetext{
(1) Aceito para publicação em 6 de agosto de 2001.

(2) Embrapa-Centro de Pesquisa Agropecuária do Trópico Semi-Árido, Caixa Postal 23, CEP 56300-970 Petrolina PE. E-mail: gmilanez@ufla.br

(3) Universidade Federal de Lavras, Dep. de Agricultura, Caixa Postal 37, CEP 37200-000 Lavras, MG. Bolsista do CNPq E-mail: rovilson@ufla.br
}

plantas, têm apresentado grande potencial de utilização na agricultura. Têm sido identificados como substâncias antigiberelínicas pelo fato da sua atuação no crescimento das plantas poder ser revertida pela aplicação de uma giberelina exógena. O paclobutrazol é um derivado do triazol, com atividade sobre o retardamento de crescimento de plantas (Graebe, 1987), cujo modo de ação consiste na inibição da biossíntese da giberelina (Steffens et al., 1985). A alta atividade das giberelinas induzindo o pseudoperfilhamento em alho (Moon \& Lee, 1980) sugere que substâncias antigiberelínicas, como o paclobutrazol, poderiam apresentar algum controle 
sobre esta anormalidade, possibilitando o uso racional de fatores de produção como o $\mathrm{Ne}$ a água.

Na cultura do alho, a utilização de hormônios retardantes de crescimento vem sendo estudada no controle do pseudoperfilhamento, assim como em outras hortaliças, no controle da altura de plantas. Foda et al. (1979) verificaram que a imersão de bulbilhos em solução de cycocel e a pulverização de plantas por duas vezes com $1.000 \mathrm{ppm}$ resultaram em maior produtividade, bulbos de maior tamanho e maior número de bulbilhos por bulbo. Silva (1984), avaliando a influência de chlormequat (1.000 ppm) e ácido giberélico (200 ppm), não constatou nenhum efeito desses tratamentos no controle do pseudoperfilhamento.

Com a aplicação de paclobutrazol em diferentes cultivares de alho, Souza (1990) observou redução na altura das plantas na cultivar Juréia e menor número de folhas na cultivar B.G.A. 8701, aumentando comercialmente a produção de bulbos das duas cultivares e reduzindo a da cultivar Amarante. O incremento das doses causou redução no peso médio de bulbos na cultivar Juréia, não se verificando efeito significativo quanto ao número de bulbilhos por bulbo. A incidência de bulbos pseudoperfilhados foi significativamente reduzida nas cultivares Juréia e B.G.A. 8701. Maior produção de bulbos graúdos e médios com a dose de 1.082 e 708 ppm, respectivamente, foi constatada por Resende (1992), com um incremento de $10,4 \%$ e $6,4 \%$ em relação à testemunha. Na produção de bulbos pequenos, verificou-se efeito quadrático e a menor produção foi obtida pela dose de 883 ppm, que causou uma redução de 14,7\%, em relação à testemunha.

Resende et al. (1993) verificaram com o incremento das doses de paclobutrazol efeito linear sobre a produção total de bulbos da cultivar de alho Quitéria, não encontrando diferenças significativas quanto a produção comercial e porcentagem de bulbos pseudoperfilhados. Rahim \& Fordham (1990) constataram que a imersão dos bulbos em paclobutrazol a $5 \mathrm{ppm}$ e cycocel a $1.000 \mathrm{ppm}$ por 24 horas antes do plantio foram efetivos para acelerar o desenvolvimento da cultura, e em algumas épocas de plantio aumentaram a produtividade de bulbos. Tais resultados permitem inferir que esses reguladores de crescimento podem ser usados para substituir a necessi- dade de frio da cultura, já que as pulverizações após o plantio não se mostraram efetivas; resultados similares foram obtidos por Rahim et al. (1994).

A produtividade total e comercial de bulbos de alho, porcentagem de bulbos pseudoperfilhados e número de bulbilhos por bulbo não foram afetados pelo uso de paclobutrazol (Resende et al., 1999). No entanto, os autores observaram efeito quadrático entre a porcentagem de bulbos graúdos e as concentrações de paclobutrazol, sendo que a concentração de $451 \mathrm{mg} \mathrm{L}^{-1}$ promoveu aumento superior a $4,5 \%$ em relação à testemunha. Houve também efeito quadrático entre a produção de bulbos médios, e as doses de paclobutrazol e a menor produção foi obtida com a concentração de $385 \mathrm{mg} \mathrm{L}^{-1}$, superando a testemunha em 5,6\%. Resultados similares foram verificados na produção de bulbos pequenos, cuja concentração de $522 \mathrm{mg} \mathrm{L}^{-1}$ propiciou a menor produção de bulbos pequenos, ou seja, $16,6 \%$ menor que a testemunha.

Resende et al. (1994) relataram que a aplicação de paclobutrazol reduziu a altura de planta aos 40 dias após o plantio, não sendo eficiente aos 80 dias e não mostrando diferenças significativas tanto no número de folhas como no número de bulbilhos por bulbo. Em outras hortaliças, como o tomate, brócolo e morango, a aplicação de paclobutrazol reduziu o crescimento das plantas (Pombo et al., 1985; Ramina et al., 1985).

O uso de retardantes de crescimento em cultivares de hortaliças tem-se tornado uma opção para controlar, sobretudo, a altura das plantas, contudo pouco se sabe sobre os efeitos do paclobutrazol nos processos fisiológicos das plantas que possam contribuir para o incremento da produtividade e controle do pseudoperfilhamento.

Este trabalho objetivou estudar o efeito de doses de paclobutrazol na produtividade e nas características morfológicas e comerciais do alho.

\section{Material e Métodos}

\footnotetext{
O experimento foi realizado de maio a outubro de 1991, no campo experimental do setor de olericultura da Universidade Federal de Lavras (Ufla), Lavras, MG, em delineamento de blocos ao acaso. Os tratamentos constituíram-se de quatro doses de paclobutrazol $(0,500,1.000$ e $1.500 \mathrm{mg}$
} 
de i.a. $\left.\mathrm{L}^{-1}\right)$, com cinco repetições. A área útil da parcela foi de $1,6 \mathrm{~m}^{2}(0,8 \mathrm{~m} \times 2,0 \mathrm{~m})$ e o espaçamento de plantio foi de $0,2 \mathrm{~m}$ entre linhas e $0,1 \mathrm{~m}$ entre plantas.

Utilizaram-se bulbos de alho da cultivar Quitéria, proveniente de Curitibanos, SC, que foram vernalizados em câmara fria por 40 dias a $5 \pm 1^{\circ} \mathrm{C}$. Os bulbilhos para plantio foram os retidos em peneira 3 (malha $8 \times 17 \mathrm{~mm}$ ), os quais foram colocados imediatamente após a vernalização em imersão por uma hora, nas diferentes doses de paclobutrazol, e secados à sombra por 24 horas.

A análise do solo antes da instalação do experimento apresentou as seguintes características químicas e granulométricas: $\mathrm{K}, 0,4 \mathrm{cmol}_{\mathrm{c}} \mathrm{dm}^{-3}, \mathrm{P}, 19 \mathrm{mg} \mathrm{dm}^{-3}$; $\mathrm{Ca}$, $3,3 \mathrm{cmol}_{\mathrm{c}} \mathrm{dm}^{-3} ; \mathrm{Mg}, \quad 0,3 \mathrm{cmol}_{\mathrm{c}} \mathrm{dm}^{-3} ; \mathrm{H}+\mathrm{Al}$, $0,1 \mathrm{cmol}_{\mathrm{c}} \mathrm{dm}^{-3} ; \mathrm{pH}$ (água), 5,8; matéria orgância, $30 \mathrm{~g} \mathrm{~kg}^{-1}$, areia, $315 \mathrm{~g} \mathrm{~kg}^{-1}$; silte, $335 \mathrm{~g} \mathrm{~kg}^{-1}$; argila, $350 \mathrm{~g} \mathrm{~kg}^{-1}$.

O preparo do solo foi feito pelo processo convencional e a adubação básica de plantio constou de $700 \mathrm{~kg}$ de superfosfato simples, $200 \mathrm{~kg}$ de cloreto de potássio, $50 \mathrm{~kg}$ de sulfato de magnésio, $10 \mathrm{~kg}$ de sulfato de zinco e $15 \mathrm{~kg}$ de bórax, por hectare (Filgueira, 1982). Aos 45 dias após o plantio aplicaram-se, em cobertura, $20 \mathrm{~kg} \mathrm{ha}^{-1}$ de nitrogênio. As irrigações por aspersão foram feitas duas vezes por semana até 20 dias antes da colheita, e a limpeza da cultura mediante capinas manuais. O controle fitossanitário foi feito semanalmente com produtos à base de maneb, para controle preventivo da alternaria, e duas pulverizações de vamidothion para controle de tripes. Não foi constatada a ocorrência de ferrugem ou outras doenças durante a realização do experimento.

Após a colheita, os bulbos permaneceram no campo por três dias, e em seguida no galpão, por 60 dias $\mathrm{Na}$ seqüência, foi cortada a parte aérea a $1 \mathrm{~cm}$ dos bulbos e retirando-se suas raízes e túnicas externas

Foram avaliadas as variáveis altura das plantas e número de folhas por planta aos 60 e 90 dias após o plantio; produtividade total e comercial (bulbos perfeitos, livres de doenças e anormalidades fisiológicas, com diâmetro superior a $25 \mathrm{~mm}$ ); peso médio dos bulbos; porcentagem de bulbos pseudoperfilhados; e número de bulbilhos por bulbo. Os efeitos dos fatores estudados sobre as características avaliadas foram conhecidos mediante a análise de variância e regressão polinomial, a 5\% de probabilidade. Os dados de porcentagem foram previamente transformados em arco seno $(\mathrm{P} / 100)^{0,5}$

\section{Resultados e Discussão}

A altura de plantas de alho foi influenciada significativamente pelas doses de paclobutrazol, verificando-se redução linear com o incremento das doses nas avaliações aos 60 e 90 dias após o plantio (Figura 1). A redução do porte da planta pelo uso desse regulador também foi verificada por Souza \& Casali (1992) e Resende et al. (1994). Outros autores trabalhando com diferentes espécies de plantas observaram redução no porte delas devido ao emprego desse regulador de crescimento (Pombo et al., 1985; Ramina et al., 1985). Com o uso de outras substâncias consideradas antigiberelínicas, que atuam de forma semelhante, Foda et al. (1979) e Souza \& Casali (1991) verificaram redução na altura de plantas de alho com o uso de chlormequat, e Tewari et al. (1984) aplicando daminozide.

Com relação ao número de folhas por planta, foram encontradas respostas similares; no entanto, observou-se que o efeito do produto foi muito pequeno comparando-se a ausência à maior dose aplicada $(4,6 \%$ e $6,7 \%$, respectivamente, nas avaliações aos 60 e 90 dias pós-plantio) (Figura 1). Souza (1990) encontrou resultados similares com a cultivar de B.G.A. 8701, salientando que o efeito do mesmo pro-
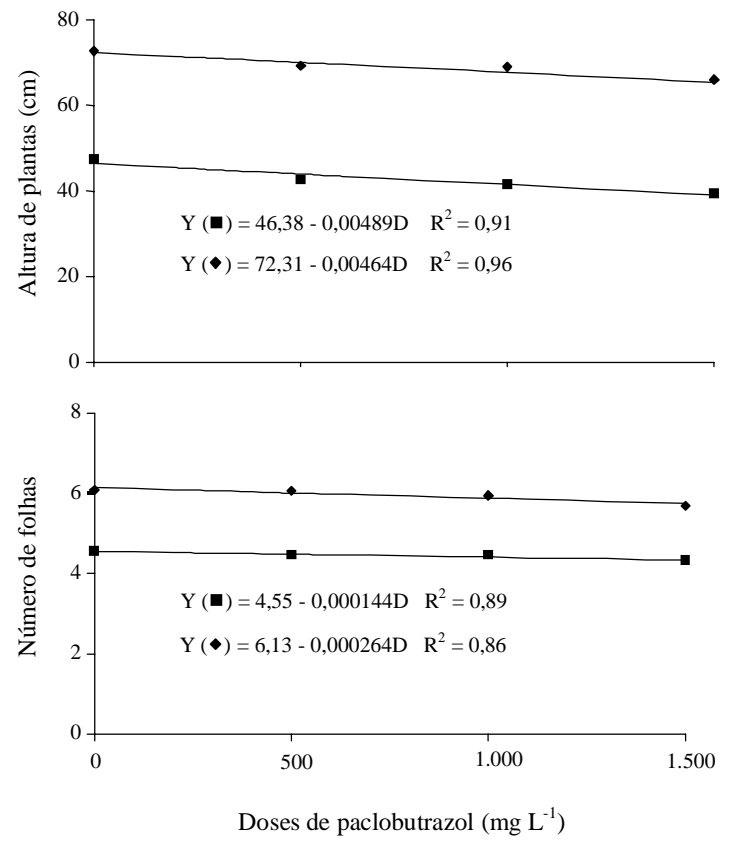

Figura 1. Altura de plantas e número de folhas de alho aos $60(\boldsymbol{\square})$ e $90(\diamond)$ dias após o plantio em razão de doses de paclobutrazol (D). 
duto foi muito pequeno, e não observou efeitos sobre a cultivar Juréia. Resende et al. (1994) não constataram efeitos de doses de paclobutrazol sobre o número de folhas na cultivar Quitéria. Como as folhas são os órgãos vegetativos responsáveis pela atividade fotossintética, seu maior número determina uma melhor produtividade. Nesse sentido, a pequena redução promovida pelo produto não ocasionou queda na produtividade, neste trabalho; contudo, a redução da altura da planta permitirá melhor arranjo populacional, o qual poderá promover maior incremento na produtividade do alho.

A produtividade total e comercial de bulbos apresentou efeito quadrático significativo em relação às doses de paclobutrazol (Figura 2). As concentrações

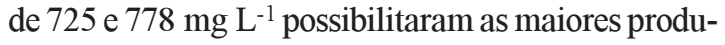
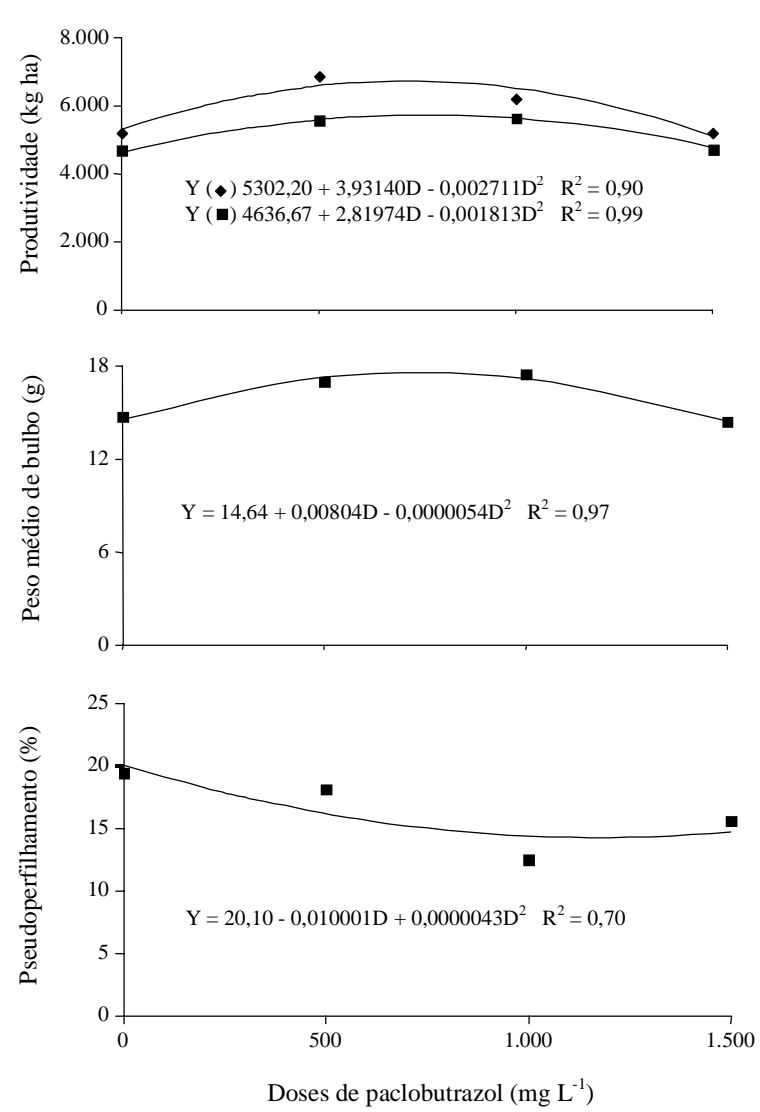

Figura 2. Produtividade total e comercial , peso médio de bulbos e porcentagem de bulbos de alho pseudoperfilhados em razão das doses de paclobutrazol (D). tividades total e comercial de bulbos de alho, respectivamente. Resultados similares são relatados por Rahim \& Fordham (1990) e Rahim et al. (1994), que observaram incrementos na produtividade de bulbos de alho, e por Souza \& Casali (1992), que também encontraram aumentos na produtividade comercial de bulbos com a aplicação de paclobutrazol, com a cultivar Juréia, assim como Resende et al. (1993) constataram maior produtividade total de bulbos utilizando a cultivar Quitéria.

A relação entre a porcentagem de bulbos pseudoperfilhados e as doses de paclobutrazol foi quadrática, e a maior redução na porcentagem de pseudoperfilhamento foi propiciada pela dose de $1.163 \mathrm{mg} \mathrm{L}^{-1}$ do produto (Figura 2). Souza (1990) observou redução de $24,0 \%$ de bulbos pseudoperfilhados quando aplicou $500 \mathrm{mg} \mathrm{L}^{-1} \mathrm{de}$ paclobutrazol, e Resende et al. (1999), mesmo não encontrando diferença significativa quanto a essa característica, salientaram que a aplicação de paclobutrazol $\left(1.500 \mathrm{mg} \mathrm{L}^{-1}\right)$ reduziu em $17,4 \% \mathrm{a}$ porcentagem de bulbos pseudoperfilhados.

A dose de $744 \mathrm{mg} \mathrm{L}^{-1}$ de paclobutrazol proporcionou o maior peso médio de bulbos (Figura 2). Resende et al. (1999) constataram maior peso médio quando aplicaram $976 \mathrm{mg} \mathrm{L}^{-1}$ do referido produto. No entanto, contradizem as afirmações de Souza (1990), que verificou redução no peso médio de bulbos com o uso de paclobutrazol, embora no nível de $10 \%$ de probabilidade.

Quanto ao número de bulbilhos por bulbo não foram verificados efeitos significativos das doses de paclobutrazol, os quais variaram de 9,8 a 11,5 bulbilhos/bulbo, corroborando Souza (1990) e Resende et al. (1994, 1999). O maior peso médio de bulbo e a ausência de resposta do paclobutrazol em aumentar o número de bulbilhos por bulbo reflete diretamente na melhoria da qualidade dos bulbos, cujas melhores cotações em nível de comercialização são obtidas pelos bulbos de maior tamanho e com pequeno número de bulbilhos por bulbo.

\section{Conclusões}

1. O paclobutrazol reduz a altura e o número de folhas de alho aos 60 e 90 dias após o plantio e a porcentagem de bulbos de alho pseudoperfilhados. 
2. O paclobutrazol proporciona aumentos na produtividade total e comercial e no peso médio dos bulbos.

3. A maior produtividade de bulbos comerciais de alho é obtida com a dose de $778 \mathrm{mg} \mathrm{L}^{-1} \mathrm{de}$ paclobutrazol.

4. O número de bulbilhos por bulbo não é afetado pelo uso de paclobutrazol.

\section{Referências}

FILGUEIRA, F. A. R. Manual de olericultura: cultura e comercialização de hortaliças. São Paulo: Agronômica Ceres, $1982.357 \mathrm{p}$

FODA, S. A.; SALEH, H. H.; SHAHEIN, A. H. Effect of cycocel "chlormequat" on garlic. Agricultural Research Review, Cairo, v. 57, n. 3, p. 171-177, 1979

GRAEBE, J. E. Gibberellin biosynthesis and control. Annual Review of Plant Physiology, Palo Alto, v. 38 , p. $419-465,1987$

MOON, W.; LEE, B. Y. Influence of short day treatment on the growth and levels of endogenous growth substances in garlic plants (Allium sativum L.). Journal of the Korean Society for Horticultural Science, Seoul, v. 21, n. 2, p. $109-118,1980$

POMBO, G.; ORZOLEK, M. D.; TUKEY, L. D. The effect of paclobutrazol, daminozide, glyphosate and 2,4-D in gel on the emergence and growth of germinated tomato seeds. Journal of Horticultural Science, Ashford, v. 60, n. 3, p. 353-367, 1985.

RAHIM, M. A.; FORDHAM, R. The advancement for bulbing in garlic (Allium sativum L.) with specific reference to late season production in Bangladesh. Acta Horticulturae, Wageningen, n. 267, p. 271-277, 1990.

RAHIM, M. A.; FORDHAM, R.; MIDMORE, D. J. Control of bulbing in garlic. Acta Horticulturae, Wageningen, n. 358, p. 369-374, 1994.

RAMINA, A.; TONUTTI, P.; TOSI, T. The effect of paclobutrazol on strawberry growth and fruiting. Journal of Horticultural Science, Ashford, v. 60, n. 4, p. 501-506, 1985
RESENDE, G. M. de. Influência do nitrogênio e paclobutrazol na cultura do alho (Allium sativum $\mathbf{L}$.) cv. "Quitéria". 1992. 107 f. Dissertação (Mestrado) - Escola Superior de Agricultura de Lavras, Lavras.

RESENDE, G. M. de; COSTA, N. D.; MELO, N. F. de; SOUZA, R. J. de. Efeitos de paclobutrazol em diferentes concentrações e períodos de imersão na cultura do alho. Pesquisa Agropecuária Brasileira, Brasília, v. 34, n. 4 , p. 635-639, abr. 1999.

RESENDE, G. M. de; SOUZA, R. J. de; LUNKES, J. A Efeito do nitrogênio e paclobutrazol sobre algumas características morfológicas e perda de peso de plantas e bulbos de alho (Allium sativum L.) cv. Quitéria. Ciência e Prática, Lavras, v. 18, n. 3, p. 274-279, jul./set. 1994.

RESENDE, G. M. de; SOUZA, R. J. de; LUNKES, J. A. Influência do nitrogênio e paclobutrazol em alho cv. Quitéria. Horticultura Brasileira, Brasília, v. 11, n. 2, p. 126-128, nov. 1993.

SILVA, N. F. D. da. Estudo da superação da dormência, crescimento e produção do alho (Allium sativum $\mathbf{L}$.) $\mathrm{cv}$. Peruano submetido a frigorificação, calor e lavagem pré-plantio e efeito de fitorreguladores na produção e nos aspectos comerciais. 1984. 86 f. Dissertação (Mestrado) - Universidade Federal de Viçosa, Viçosa.

SOUZA, R. J. de. Influência do nitrogênio, potássio, cycocel e paclobutrazol na cultura do alho (Allium sativum L.). 1990. 143 f. Tese (Doutorado) - Universidade Federal de Viçosa, Viçosa.

SOUZA, R. J. de; CASALI, V. W. D. Influência do nitrogênio e cycocel na cultura do alho (Allium sativum L.) Ciência e Prática, Lavras, v. 15, n. 1, p. 69-78, jan./mar. 1991.

SOUZA, R. J. de; CASALI, V. W. D. Influência do paclobutrazol na cultura do alho (Allium sativum L.). Ciência e Prática, Lavras, v. 16, n. 2, p. 246-251, abr.jun. 1992.

STEFFENS, G. L.; BYUN, J. K.; WANG, S. Y. Controlling plant growth via the gibberellin biosynthesis system. I: Growth parameter alterations in apple seedlings. Physiologia Plantarum, Copenhagen, v. 63, n. 2, p. 163-168, 1985.

TEWARI, J. P.; AWASTHI, D. N.; KANAUJIA, J. P.; JOSHI, K. R. Effect of growth retardants on the growth and yield of single clove garlic. Progressive Horticulture, Lucknow, v. 16, n. 3/4, p. 199-201, 1984. 